\title{
Pregnancy in women with congenital heart disease
}

\section{Rachael Hatton and Candice K Silversides}

With advances in pediatric cardiac surgery, there have been major improvements in the survival of children born with congenital heart disease (CHD). As a consequence, there is a growing population of young women with $\mathrm{CHD}$, the majority of whom will become pregnant. However, for women with CHD, pregnancy imposes a hemodynamic stress on the heart and this can result in pregnancy complications for women and their babies. Although there have been a large number of studies examining adverse pregnancy outcomes in women with CHD, some important questions remain unanswered. For instance, the true maternal mortality risk in women with CHD is not known. This is in part because maternal deaths are rare, few large cohorts are available and because there can be a survivor bias in published series. Rates of maternal cardiac complications, such as arrhythmias or heart failure, are also frequently biased as studies often include women followed in tertiary or quaternary referral centres and not those women followed in smaller hospitals. The impact of CHD on other aspects of care such as length of stay or hospital costs is rarely reported, but is important consideration for women, their families and hospital administrators. The study by Opotowsky et al. ${ }^{1}$ examines the epidemiology of cardiovascular events at the time of delivery in women with CHD and addresses many of these unanswered questions.

Opotowsky et al. ${ }^{1}$ report on maternal morbidity and mortality in a large population of women with CHD. Using the largest all-payer national administrative hospital discharge database in the United States, cardiovascular events during admission for childbirth between 1998 and 2007 were examined. CHD diagnosis was based on International Classification of Diseases 9th Revision (ICD-9) coding and lesion severity was categorized as simple, moderate, complex ${ }^{2}$ or unclassified. During the study period, there were 42,602,106 deliveries of which 30,500 were in women with CHD. The annual number of deliveries in women with and without CHD increased by $34.9 \%$ and $21.3 \%$, respectively. Maternal mortality at the time of delivery was significantly higher in women with CHD compared to those without CHD [Odd ratio (OR) 6.7, 95\% confidence interval (CI) 2.9-15.4]. Adverse maternal cardiovascular outcomes were also more common in women with CHD (OR 8.4, 95\% CI 7.0-10.0) of which arrhythmias were the most common. Supraventricular arrhythmias were the most common type of arrhythmia accounting for more than $75 \%$ of the reported arrhythmias. Women with CHD were also at higher risk for heart failure (OR 8.0, 95\% CI 6.7-10.1) and cerebrovascular accidents (OR 41.6, 95\% CI 25.8-67.1) at the time of delivery. Women with complex CHD or pulmonary hypertension, regardless of disease complexity, were at highest risk of adverse cardiovascular events during delivery. Caesarean deliveries were more common in women with CHD $(32.2 \%$ versus $26.5 \%, p<0.0001)$. Length of stay and hospital costs were longer in women with CHD compared to the general population (3.5 versus 2.6 days, $p<0.0001$ and $\$ 11,505$ versus $\$ 8,006$, $p<0.0001)$. Deliveries complicated by adverse events in women with CHD were associated with further increases length of stay (5.9 versus 3.4 days, $p<0.0001)$ and total charges $(\$ 24,888$ versus $\$ 10,935$, $p<0.0001$ ).

This study highlights the growing number of deliveries, the associated risk and the implications for health care for women with CHD. The increasing prevalence of women with CHD admitted for childbirth is consistent with studies recognizing the growing number of adults with CHD in general. ${ }^{3}$ Similar to other studies of cardiovascular outcomes during pregnancy in women with $\mathrm{CHD}$, arrhythmias and heart failure are the main complications that occur in this population and, although rare, maternal mortality is increased when compared to women without CHD. Women with complex cardiac lesions and pulmonary hypertension are at particularly high risk for adverse outcomes and these are important considerations during preconception counseling. Despite advances in medical therapies, severe pulmonary hypertension from any cause still carries a high mortality risk and pregnancy is contraindicated. ${ }^{4,5}$ While lesion complexity and pulmonary hypertension are risk factors for adverse outcomes at the time of delivery, there are a number of additional risk factors that can also help to identify women at risk and these should also should be integrated into risk assessment. ${ }^{6,7}$

In addition to maternal morbidity and mortality, other aspects of care such as length of stay and hospital costs are also increased in women with CHD, particularly in those women who develop complications at the time of delivery. Obstetric care also differs in women with CHD. Current recommendations suggest that vaginal delivery can be safely undertaken by most women with CHD. ${ }^{8,9}$ However, Caesarean deliveries which are known to be associated with higher complications rates when compared to vaginal deliveries, are more common in this group of women. The reasons for increased Caesarean deliveries in women with CHD is not explained by this study, but may reflect concern of health care providers to allow women with CHD to undergo a vaginal delivery. Further education of health care providers may be required.

Some maternal complications may be preventable. The most recent report of the Confidential Inquiries into Maternal Mortality in the United Kingdom concluded that a substantial portion of maternal cardiac deaths in pregnancy were related to substandard delivery of care. ${ }^{10}$ Care of pregnant woman with CHD is complex, requiring a multidisciplinary team of cardiologists, high-risk obstetricians, obstetric anesthesiologists, neonatologists, and nurses. Efforts to improve coordination of care and delivery of care are potential targets for quality and safety initiatives that may ultimately help to decrease adverse event rates or shorten length of stay.

\section{Acknowledgements}

Dr. R. Hatton was the recipient of the Gordon B. Allan Fellowship in Adult Congenital Heart Disease at the Toronto Congenital Cardiac Centre for Adults, Toronto, Ontario.

\section{References}

1. Opotowsky AR, Siddiqi OK, D’Souza B, et al. Maternal cardiovascular events during childbirth among women with congenital heart disease. Heart 2012; 98: 145-151.

Division of Cardiology, Obstetric Medicine Program, University of Toronto, Mount Sinai Hospital and University Health Network, Toronto, Canada

\section{Corresponding author:}

Candice K Silversides, Mount Sinai Hospital, OPG Building, 700 University Ave, 3rd Floor, Room 3-912 Toronto, ON, Canada, M5G IZ5.

Email: csilversides@mtsinai.on.ca 
2. Warnes CA, Liberthson R, Danielson GK, et al. Task force 1: the changing profile of congenital heart disease in adult life. $J$ Am Coll Cardiol 2001; 37: 1170-1175.

3. Marelli AJ, Mackie AS, Ionescu-Ittu R, et al. Congenital heart disease in the general population: changing prevalence and age distribution. Circulation 2007; 115: 163-172.

4. Bedard E, Dimopoulos K and Gatzoulis MA. Has there been any progress made on pregnancy outcomes among women with pulmonary arterial hypertension? Eur Heart $J$ 2009; 30: 256-265.

5. Kiely DG, Condliffe R, Webster V, et al. Improved survival in pregnancy and pulmonary hypertension using a multiprofessional approach. BJOG 2010; 117: 565-574.

6. Siu SC, Sermer M, Colman JM, et al. Prospective multicenter study of pregnancy outcomes in women with heart disease. Circulation 2001; 104: 515-521.
7. Drenthen W, Boersma E, Balci A, et al. Predictors of pregnancy complications in women with congenital heart disease. Eur Heart $J$ 2010; 31: 2124-2132.

8. Regitz-Zagrosek V, Blomstrom Lundqvist C, Borghi C, et al. ESC guidelines on the management of cardiovascular diseases during pregnancy: the task force on the management of cardiovascular diseases during pregnancy of the European Society of Cardiology (ESC). Eur Heart $J$ 2011; 32: 3147-3197.

9. Robertson JE, Silversides CK, Mah ML, et al. A contemporary approach to the obstetric management of women with heart disease. J Obstet Gynaecol Can 2012; 34: 812-819.

10. Cantwell R, Clutton-Brock T, Cooper G, et al. Saving mothers' lives: reviewing maternal deaths to make motherhood safer: 20062008. The eighth report of the confidential enquiries into maternal deaths in the United Kingdom. BJOG 2011; 118: 1-203. 